\title{
The DVB-T-Based Positioning System and Single Frequency Network Offset Estimation
}

\author{
Rostislav KARASEK ${ }^{1}$, Frantisek VEJRAZKA ${ }^{2}$ \\ ${ }^{1}$ Dept. of Electromagnetic Field, Faculty of Electrical Engineering, Czech Technical University in Prague, \\ Technicka 2, 16627 Prague, Czech Republic \\ 2 Dept. of Radio Engineering, Faculty of Electrical Engineering, Czech Technical University in Prague, \\ Technicka 2, 16627 Prague, Czech Republic
}

Rostislav.Karasek@fel.cvut.cz, Vejrazka@ fel.cvut.cz

Submitted April 28, 2018 / Accepted September 10, 2018

\begin{abstract}
As position information becomes more and more important in many fields of technology it is advantageous to recognize it in scenarios where satellite-based systems fail. Such a case is the scenario inside buildings where attenuation of a signal is too high making it impossible to receive despite the availability of terrestrial services. A positioning system based on terrestrial broadcasting is presented in this paper. The aim is to create an automatic receiver enabling a multi-sensor positioning system to be built and resulting in increased availability and reliability of position information. This paper introduces a method that demonstrates how to design a signal detector capable of operating in a multipath scenario. Finally, the most restrictive problem of the positioning system is the unknown time offset setting of individual emitters that render this system useless. A solution to this problem is proposed and tested in a real scenario. The innovative methods and algorithms presented in this paper show, for the first time, how to automatically evaluate position using digital video broadcasting. The result of an experiment with a real digital video broadcasting network is presented.
\end{abstract}

\section{Keywords}

Signals of Opportunity, positioning, digital video broadcasting, DVB-T, OFDM, TDoA, mismatched filtration, CA-CFAR

\section{Introduction}

Modern positioning techniques are mainly based on Global Navigation Satellite Systems (GNSS) such as Global Positioning System (GPS), Globalnaya Navigatsionnaya Sputnikovaya Sistema (GLONASS), GALILEO and BeiDou. Despite their global coverage and high precision, these positioning techniques can only be used in ideal conditions in the absence of obstacles in the line of sight between satellites and a receiver, and without a multipath channel. Creating these conditions in urban areas is problematic. Signal distortion can rapidly lower precision, and position service outage occurs frequently. Low range positioning systems can be used in these hard conditions to provide comparable precision to GNSS. For example, the Ultra-WideBand (UWB) is a modern solution for indoor navigation that provides high precision at small distances. Currently, there is no positioning system capable of indoor navigation within larger areas with the main constraint being the low signal level given by frequency allocations. More information on multipath and other types of GPS sources of error can be found in [1].

Signal power limitation begs the question of whether transmission services, such as Long Term Evolution (LTE), Digital Audio Broadcasting (DAB) and Digital Video Broadcasting - Terrestrial (DVB-T), could be used for positioning as they have similar properties as the GNSS signal. These systems use Orthogonal Frequency Division Multiplexing (OFDM) modulation, providing high time resolution necessary for positioning precision. The systems also consider positions evaluated according to signal propagation time Time of Arrival (ToA) or its difference Time Difference of Arrival (TDoA).

The DVB-T and its successor the Digital Video Broadcasting - Terrestrial 2 (DVB-T2) are promising systems which can serve as supplementary positioning systems. The transmitting power is high enough to allow good indoor reception kilometers from a transmitter. The transmission has a precise time synchronization and a high-frequency bandwidth. The propagation loss is relatively low due to the maximal frequency used in DVB-T system. Originally up to $862 \mathrm{MHz}$, now even less for mobile broadband communication. Unlike the GNSS, the Doppler effect can be neglected when DVB-T is used because of low frequency and transmitter stationarity. Those qualities make it a potential solution for an indoor positioning system with a high range. The fact that DVB-T and DVB-T2 standards are the most widelyused for a video broadcasting in Europe, Africa, Asia (except China) and Australia is another plus. 
This work is aimed to the DVB-T standard, but since the DVB-T should be replaced by DVB-T2 in following years, we need to shortly compare both systems to decide if methods used in this work will be possible in the future. Both systems are specified by ETSI standards [2] and [3]. The main differences which influence the functionality of the presented positioning system are described, and a possible solution is suggested.

A DVB-T-based positioning system has certain features that can lower the precision of the system or make it impossible to estimate position. For example, in an urban area, precision can be significantly affected by signal delays caused by propagating through an unknown number of obstacles with unidentified electric parameters. The most limiting problem is the DVB-T signal structure itself as the positioning using DVB-T is based on the Single Frequency Network (SFN) where more than one transmitter transmits the same signal at the same time with the same frequency [4], [5]. This is making impossible to identify individual transmitters. The Cell ID is only an optional parameter, and it is not typically used. This can cause position ambiguities as the received signal may be incorrectly assigned to transmitters. A possible solution to solve this problem is presented in [6].

A method how to use DVB-T broadcast for positioning has already been published in [7], [8] and [9]. The method, which performs a cross-correlation of the received signal with its ideal replica using only the pilot sequence used in [7], extracts the ToA of a signal propagated from several synchronized transmitters. This method is not available in a common scenario where only two transmitters working in SFN are used, but three emitters are necessary for 2D ToA positioning. Analysis of tracking precision using this method is published in [10], but it is necessary to include the influence of transmitter geometry to obtain the precision of a positioning system based on DVB-T. The derived ideal cross-correlation function is a cardinal sine function (sinc). Thus sidelobe separation is approximately $13 \mathrm{~dB}$. The low sidelobe separation can cause problems with the automatic signal detection as the algorithm may not be able to distinguish between the maximum corresponding to a weak signal with a nearly the same time of arrival from its sidelobe.

Suppressing the sidelobes by mismatched filtration offers a solution to the aforementioned problems. This paper presents a method that slightly extends the main lobe of the correlation function while significantly suppressing correlation sidelobes. The problem related to an insufficient number of transmitters in the SFN can be solved by using more than one SFN and fusing them. Though it is no longer possible to use a ToA positioning method, a TDoA method, which can evaluate position in such a scenario, is available. The implementation of an iterative TDoA positioning algorithm is published in [8] where information regarding the influence of transmitter placing on the value of Dilution of Precision (DoP) can also be found.

The system presented in this paper is innovative because it is capable of providing automatic positioning without any user interaction. To do so, an automatic detection algorithm is mandatory. Such a detector is presented in this paper, and it has been tested in a real multipath scenario. More information can be found in [9]. This method is based on a radar technique method called Cell Averaging - Constant False Alarm Rate (CA-CFAR) [11].

During the experiment with a real DVB-T transmission, it has been found that a constant time offset can be and usually is set to the DVB-T transmitter. This causes an error in the evaluated position. The relative offset measurement method was developed to correct this error. It estimates relative offsets in the SFN and uses them as a correction for detected time arrival differences.

The paper is divided into sections describing the necessary steps for positioning in a DVB-T network. The DVB-T signal is briefly described in Sec. 2 and attention is paid to the pilot sequence structure defined by standard [2]. The main differences between the DVB-T and DVB-T2 are shortly described in Sec. 9. The time and frequency synchronization procedure has been implemented according to [12] and is described in Sec. 3. Signal preprocessing, which improves detection properties, is presented in Sec. 4. When preprocessing is finished, it is possible to proceed to the automatic detector which is introduced in Sec. 5. The method, which solves the transmitter time offset problem in the SFN, is proposed in Sec. 6. The concept of the positioning algorithm is based on [8] and described in Sec. 7. Section 8 describes outdoor experiments performed with a real DVB-T network broadcasting. The paper concludes in Sec. 10.

\section{DVB-T Signal Properties}

The DVB-T is an OFDM-modulated signal given by [2]. Usually, the $8 \mathrm{k}$ mode, with 8,192 subcarriers, is used. Practically speaking, only 6,817 of them contain nonzero values. The duration of one DVB-T symbol is $896 \mu$ s. Each OFDM subcarrier is modulated using Binary-Phase Shift Keying (BPSK) or Quadrature Amplitude Modulation (QAM) if the subcarrier contains pilot or payload data, respectively. If more than one transmitter in the site is transmitting with the same carrier frequency, the SFN is applied with GPS time synchronization according to [4]. A so-called Mega-frame Initialization Packet (MIP) inserter is used to ensure standard fulfillment.

For positioning, we are only interested in the pilot sequence because it is predefined and does not change, unlike the random payload data. For this reason, an inspection of its main properties is required. The picked bits of the so-called Pseudo-Random Binary Sequence (PRBS) are mapped to the individual pilot subcarriers. The PRBS is based on a pseudo-random generator with similar correlative properties as the Coarse / Acquisition (C/A) ranging code in GPS. The PRBS cyclic autocorrelation function is 2,047 chips long with a correlation maximum 2,047, and -1 elsewhere, as shown in Fig. 1. 
For comparison, the C/A Pseudo-Random Noise (PRN) code cyclic autocorrelation length is 1023 chips long and with maximal value 1023 . The $\mathrm{C} / \mathrm{A}$ cyclic autocorrelation function acquires values 1023, 63, -1, -65 as shown in Fig. 2.

GPS chips are mapped using time division, unlike the PRBS chips, which are mapped using frequency division of a selected set of chips. This presents a problem and explains why the cyclic Autocorrelation Function (ACF) of the PRBS is not suitable for studying positioning properties. Consequently, the ACF of the actual pilot signal, which shows the necessary time domain characteristic for positioning, must be used.

The cyclic ACF of the DVB-T pilot signal can be easily derived from Fast Fourier Transform (FFT) properties. First, we need to show how to create the DVB-T pilot replica to evaluate its ACF. The OFDM modulation is defined by:

$$
s(t)=\sum_{n} \mathbf{q}_{n}^{\mathrm{T}} \mathbf{g}\left(t-n T_{\mathrm{S}}\right)
$$

where $\mathbf{q}_{n}$ is a vector of data symbols in the $n^{\text {th }}$ OFDM symbol and $\mathbf{g}\left(t-n T_{\mathrm{S}}\right)$ is the so-called expansion part of the modulation. The symbol duration is given by $T_{\mathrm{S}}$. It contains a vector of the modulation pulses corresponding to the individual subcarriers. The Discrete Fourier Transform (DFT) implementation of the OFDM modulation is, for all intents and purposes, the only method used. The modulation simply becomes the application of the inverse DFT to the vector of the data symbols. This produces a complex envelope of the DVB-T signal which is modulated to the desired carrier frequency.

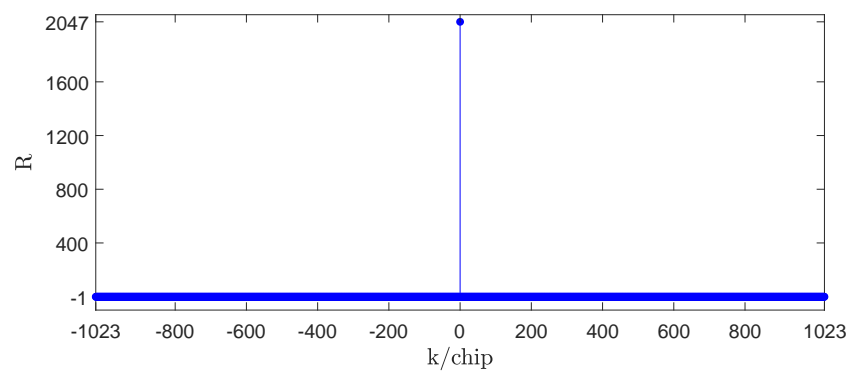

Fig. 1. One period of the PRBS cyclic autocorrelation function.

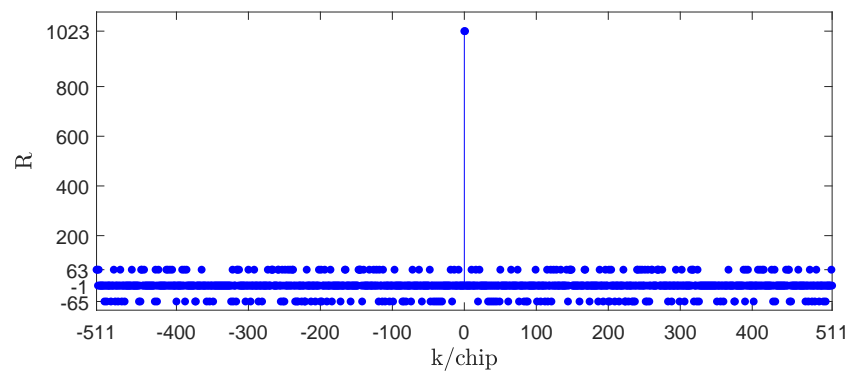

Fig. 2. One period of the C/A ranging code cyclic autocorrelation function.
The pilot sequence consists of three parts defined by [2]. Transmission Parameter Signalling (TPS) symbols are mapped to the same predefined subcarriers in each OFDM symbol and contain information related to the transmission scheme. The Continual Pilot (CP) is also mapped to the same predefined subcarriers in every OFDM symbol, but no information is inserted. Scattered Pilot (SP) data are not mapped to the same subcarriers in every OFDM symbol. One SP bit is in every $12^{\text {th }}$ subcarrier according to:

$$
\begin{aligned}
\mathbf{S P} & =3 \times(l \bmod (4))+12 \mathbf{p} \\
\mathbf{p} & =<0 ; 567>; \mathbf{p} \in \mathbb{Z}
\end{aligned}
$$

where vector SP consists of SP subcarrier numbers and $l$ is the OFDM symbol number. Since SP repeats itself every four OFDM symbols, it is not necessary to know the absolute value of $l$ to obtain the SP sequence. If we wish to create a pilot signal replica, we need to obtain the actual value of $l$ mod (4) which can be obtained using the algorithm described in [13]. A similar method is also used in [7] for the same reason. This algorithm can be described by:

$$
\begin{array}{r}
l=\arg \max _{m} \mid \sum_{p=0}^{567}\left(R_{l, 12 p+3 m} P_{12 p+3 m}\right) \\
\left(R_{l-1,12 p+3(m-1)}^{*} P_{12 p+3(m-1)}^{*}\right) \mid
\end{array}
$$

where $R_{l, 12 p}$ is the value on the $(12 p)^{\text {th }}$ subcarrier of the $l^{\text {th }}$ received OFDM symbol. $P_{12 p+3 m}$ contains the $(12 p+3 m)^{\text {th }}$ value of a PRBS. The obtained value $l$ is then applied to generate the SP sequence according to (2). This algorithm tests four possible patterns of the SP sequence and compares values at those subcarriers in actual and previous OFDM symbol.

Having obtained the actual SP sequence, we can evaluate cyclic ACF by multiplying the spectrum of the signal, with its complex conjugated replica, using a Fourier transform identity. Since the SP sequence consists only of values 1 and -1 , multiplying the spectrum of the signal sets all the pilot subcarriers to 1 . Only the pilot subcarriers are selected. This leads to a sequence of ones which have a Dirac delta DFT identity which is convenient for ACF estimation. This also, inevitably, constrains the duration of the ACF. The DVB-T symbol duration is $896 \mu$ s but only every twelfth subcarrier contains the SP symbol which can be used for ACF estimation, thus the resulting duration is $74 . \overline{6} \mu \mathrm{s}$.

The duration of the SP sequence leads to limitations of the DVB-T based positioning system. The propagation time of the signal from one transmitter to any other transmitter in this SFN has to be less than a half of the autocorrelation repetitive period $(74 . \overline{6} \mu \mathrm{s})$. Otherwise, ambiguities can occur. 


\section{DVB-T Signal Synchronization}

Before the DVB-T signal can be further processed, it is necessary to perform time and frequency synchronization. This section briefly describes how to find the beginning of the OFDM symbol and frequency offset. When the OFDM system is involved, the method described in [12], using the Maximum Likelihood (ML) estimator, is usually accepted for this purpose.

The first necessary step is to choose an appropriate sampling frequency. Symbol duration is $T_{U}=896 \mu$ s, and $\frac{1}{T_{\mathrm{U}}}$ gives the frequency spacing of subcarriers in the OFDM systems. So, in DVB-T if the sampling frequency is an integer $\mathrm{MHz}$, the integer number of subcarriers in the sampled signal is ensured. This fact is convenient for any standard radio front end with a fixed sampling frequency. If we assume an integer $\mathrm{MHz}$ sampling frequency, we need to use at least $9 \mathrm{MHz}$ to sample the DVB-T broadcast correctly.

The OFDM modulation normally uses a so-called guard interval to ensure the cyclic character of the transmitted signal $s(t)$. Then, the receiver is able to use the DFT-based demodulation with the suppressed Inter-Symbol Interference (ISI). The guard interval is a cyclic continuation of the actual OFDM symbol and this guard interval will be used for time and frequency synchronization using ML function $\gamma(\tau)$ as described in [12]. Since its duration in DVB-T can vary, it is necessary to estimate Guard Interval Duration $\left(T_{\Delta}\right)$ to separate the sampled signal into individual OFDM symbols. For this reason, the algorithm described in [8] is used. This method is defined by:

$$
\begin{aligned}
\gamma(\tau) & =\int_{0}^{T_{\Delta \mathrm{MAX}}} s(t-\tau) \cdot s^{*}\left(t-\tau-T_{\mathrm{U}}\right) \mathrm{d} t \\
\gamma^{\prime}(\tau) & =\frac{\mathrm{d} \gamma(\tau)}{\mathrm{d} \tau}, \\
T_{\Delta} & =\max _{\tau}\left(\gamma^{\prime}(\tau)\right)
\end{aligned}
$$

where $\gamma(\tau)$ is a likelihood function similar to the maximum likelihood used in [12]. The main difference is that the $T_{\triangle \mathrm{MAX}}$ is not set to the actual guard interval duration value. The maximal possible guard interval duration is used instead. If the signal is normalized, then the maximum of the likelihood function derivation is the estimated $T_{\Delta}$. The guard interval duration in the SFN is usually fixed, but it can be changed. This is why it is necessary to use this algorithm for initialization.

When the guard interval duration is known, the time offset $\theta$ and the frequency offset $\varepsilon$ can be found using a likelihood function according to [12] defined by:

$$
\begin{aligned}
\gamma(\tau) & =\int_{0}^{T_{\Delta}} s(t-\tau) \cdot s^{*}\left(t-\tau-T_{\mathrm{U}}\right) \mathrm{d} t, \\
\theta & =\arg \max _{\tau} \gamma(\tau), \\
\varepsilon & =\angle \max _{\tau} \gamma(\tau) .
\end{aligned}
$$

This synchronization method gives only a rough time offset value, and it is not possible to demodulate the signal without a phase error of data points in the constellation diagram. However, the precision is sufficient to obtain the arrival time differences of the signal necessary for positioning.

\section{Mismatched Filtration}

After the successful synchronization of the signal, we can proceed to mismatched filtration. This method, which uses a windowed signal replica as an impulse response of a filter applied on the received signal and is employed for detection in radar theory [11], suppresses sidelobes causing false detections.

The filtration is generally described by a convolution according to:

$$
r(\tau)=\int_{-\infty}^{+\infty} s(t) h(t-\tau) \mathrm{d} \tau
$$

where $h(t)$ is the impulse response of a filter. We can define matched filtration as a convolution of the signal with the impulse response of the matched filter, which can be generated from an ideal replica of the signal by temporal axis inversion and complex conjugation. It is necessary to filter the matched filter with the impulse response of a window function to obtain a mismatched filter. In this case, the used ideal signal is based on an actual SP signal obtained according to the method described in Sec. 2.

The mismatched filter is realized using a Taylor Window with two nearly constant-level sidelobes and a peak sidelobe level of $-23 \mathrm{~dB}$, though it is possible to use another window type. The properties of window functions are described in [14]. The difference between matched filtration and the mismatched filtration is shown in Fig. 3. The main lobe of the matched filter is narrower than the mismatched, but since the spectral characteristic is rectangular, the sidelobe level is only $-13 \mathrm{~dB}$.

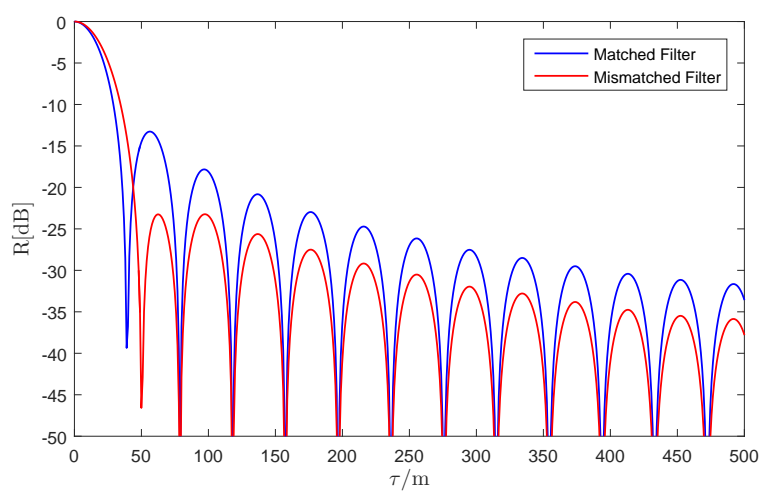

Fig. 3. A comparison of the matched filter and mismatched filter correlation function 


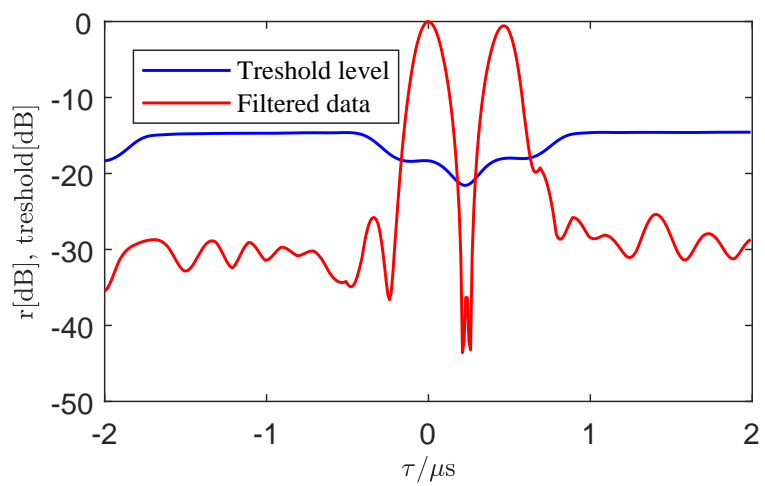

Fig. 4. The detection algorithm output for two poorly separated signals using mismatched filtration.

The properties of the automatic detection algorithm are improved thanks to the mismatched filtration. The mismatched filtration allows distinguishing closely separated signal replicas from its sidelobes. Figure 4, which is illustrative of this improvement, shows a detail of the processed measurement performed at the Strahov $\left(\mathbf{R x}_{2}\right)$ location. The time of arrival from two transmitters is nearly the same in this scenario. So, we need to distinguish between two neighboring maxims to evaluate the position. The threshold is exceeded twice. Hence two detections are indicated. The method, how the threshold level is obtained, is explained in the following section.

The small peak on the left side, with magnitude at approximately $-25 \mathrm{~dB}$, is the sidelobe of the first detection and it has been correctly deduced that it is not a signal replica since this peak is lower than the threshold level. If the mismatched filter was not used, the amplitude would be $-13 \mathrm{~dB}$ and it could cause a false detection. It would be necessary to increase the threshold near the main lobe and cause the detector to be blind for peaks which are undesirably close together. The mismatched filtration proves to be the correct way to suppress false detections caused by sidelobes while accurately detecting tightly separated signal replicas.

\section{Automatic Detection Algorithm}

The key step for positioning is to extract TDoA from the received DVB-T signal filtered by the mismatched filter. The extracted time differences are the input values for the TDoA positioning algorithm. The CA-CFAR algorithm [11] is utilized as an automatic DVB-T signal detector. The algorithm automatically chooses the threshold $(\gamma)$, according to the actual signal level, to maximize Probability of Detection $\left(P_{\mathrm{D}}\right)$ with constant Probability of False Alarm $\left(P_{\mathrm{FA}}\right)$ using the Neyman-Pearson theorem [15] given by:

$$
L(x)=\frac{p\left(x ; \mathcal{H}_{1}\right)}{p\left(x ; \mathcal{H}_{0}\right)}>\gamma
$$

where $\mathcal{H}_{0}$ and $\mathcal{H}_{1}$ are null and alternative hypothesis respectively, and the threshold $(\gamma)$ can be obtained from:

$$
P_{\mathrm{FA}}=\int_{x: L(x)>\gamma} p\left(x ; \mathcal{H}_{0}\right) \mathrm{d} x
$$

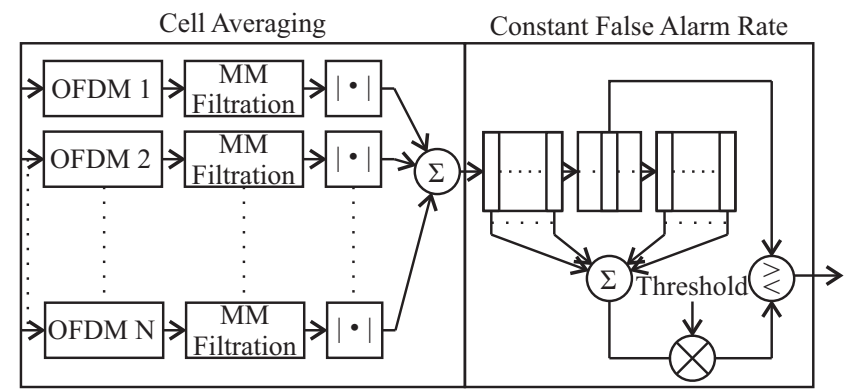

Fig. 5. Principle of the CA-CFAR algorithm used for detection of the delayed DVB-T pilot signal replicas.

In the CA-CFAR algorithm, the threshold is adapted according to the averaged output of the mismatched filter and then compared to see if the actual value exceeds the estimated threshold to achieve maximal $P_{\mathrm{D}}$ for given $P_{\mathrm{FA}}$. The principle of the algorithm is shown in Fig. 5. The DVB-T signal is separated into individual OFDM symbols, each of which is filtered using a corresponding mismatched (MM) filter. Bank of four different filters, one for each SP sequence, is created.

Then, the absolute value of the mismatched filter output is used for the next processing. The method, using the absolute value of the filtered signal, is called a noncoherent integrator. In a radar terminology, the integrator is a label for a technique realizing the averaged received pulse to enhance Signal-to-Noise Ratio (SNR) properties.

A comparison of the used integration methods can be found in [11]. The best performance can be obtained using a coherent integrator which averages individual pulses including phase. This would require nearly perfect synchronization of each received OFDM symbol. In the other case, symbols would be out of phase, and their summation could cause a signal to be neglected. The non-coherent integrator is used because of its lower synchronization sensitivity. In this case, the individual OFDM symbols are added together using absolute value. Unfortunately, a high number of averaged pulses is required to obtain the same $P_{\mathrm{D}}$. On the other hand, the synchronizer can be simplified to allow faster processing and can process more OFDM symbols in a real-time than if a coherent integrator with a precise synchronizer is used.

It is necessary to mention that if the entire OFDM symbol is filtered using a mismatched filter, then aliasing occurs in the output and causes false detections. This effect is a direct consequence of the SP sequence duration $(74 . \overline{6} \mu \mathrm{s})$. Thus, the cyclic filtration is implemented and performed only for a slightly shorter period than the sequence duration. This also significantly lowers computation complexity. Unfortunately, ambiguities occur because the output detections are of $\bmod (74 . \overline{6}) \mu$ s. This ambiguity can be solved by using additive information. Since the system is based as a supplementary system for so-called hard condition positioning, it is possible to assume that the initial position is obtained from another system and it is used to solve ambiguities to match each detection to the corresponding DVB-T transmitter. After the loss of the primary ranging signal, the system continues tracking using only the DVB-T. 
The averaged signal is then processed by the core of the CA-CFAR algorithm. It is a shift register divided into three windows with a different number of memory cells. The content of the left and right window is added together to obtain the mean level of the signal. This value is multiplied by the threshold constant and used as a threshold level. The number of cells in these windows should be set up to be long enough to include signal multipath, but not too long to include a signal from a previous or following transmitter in the SFN. The middle window represents the guard interval, and it is omitted from the threshold level estimation. The number of cells should be long enough to cover the main lobe of the filtered signal. If the value in the guard window exceeds the estimated threshold, detection occurs. All obtained detections are saved and then sent to the positioning algorithm.

\section{Single Frequency Network Offset Measurement}

The last problem to solve before the position can be evaluated is the estimation of the offset in the SFN. This problem was detected during the measurements of the DVB-T network in Prague (Czech Republic). Time differences obtained from detections in the measured signal in a known position did not correspond with the geometrical layout of the performed measurement even if $\bmod (74 . \overline{6}) \mu$ s was assumed. Similar results were obtained for every observation with the same receiver position. The explanation of this effect is that the DVB-T transmitters are synchronized according to [4], but the constant time delay between the synchronized time and the actual start of transmission is present. A method for the SFN time offset estimation is presented in this section.

The time offsets in the SFN cause an effect which can be interpreted as an increase of the transmitter distance from the receiver position. This is shown in Fig. 6 where the SFN, with two receivers in positions given by vector $\mathbf{R} \mathbf{x}_{\mathbf{n}}$, and two transmitters, placed in $\mathbf{T} \mathbf{x}_{\mathbf{0}}$, is utilized. The distance between them is given by the speed of light and the signal propagation time $\left(\mathrm{c} t_{n o}\right)$. The virtual prolonging of the receiver to transmitter distance caused by the time offset is $\mathrm{c} \Delta_{o}$.

It is more important to show how this phenomenon influences detections, as illustrated in Fig. 7. We define the output of the detection algorithm as a matrix $\mathbf{D}$ with elements $D_{\text {npo }}$ given by:

$$
D_{\text {npo }}=-D_{\text {nop }}=\tau_{n p}-\tau_{n o}
$$

where receivers are indexed by $n$ and transmitters by $o$ and $p$.

If the offsets $\Delta_{o}$ are known it is possible to evaluate the signal propagation time $t_{n o}$ from pseudo-propagation time $\tau_{n o}$ as a $\tau_{n o}=t_{n o}+\Delta_{o}$ and obtain ideal time differences given by matrix $\mathbf{T}$ with the elements:

$$
T_{n p o}=-T_{n o p}=t_{n p}-t_{n o} .
$$

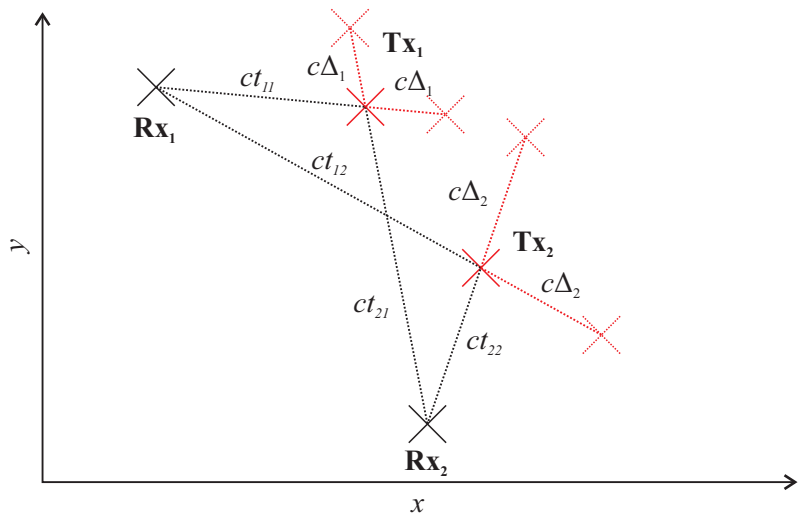

Fig. 6. The SFN time offset effect illustration.
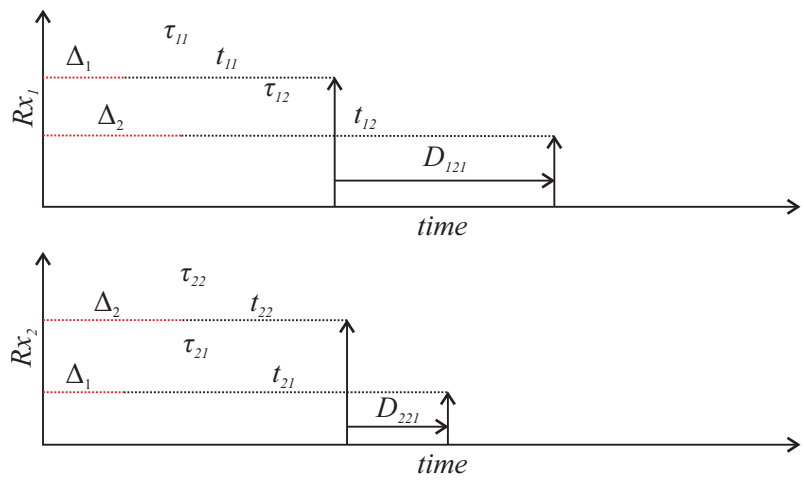

Fig. 7. The SFN time offset effect illustration in filtered data.

Since the receiver time is relative, it is not possible to measure the transmitters' time offset, but only the differences between them. Those time offset differences are organized in matrix $\mathbf{O}$ with elements:

$$
O_{p o}=-O_{o p}=\Delta_{p}-\Delta_{o} .
$$

Now, it is more obvious how to estimate a correction for a positioning algorithm with this definition of a problem. For a given network, in which we know the transmitters' coordinates, we can perform measurements on known positions, which allow us to identify $\mathbf{T}$. The detection matrix is obtained from the measured data. Then, the offset matrix is evaluated as an $\mathbf{O}=\mathbf{D}-\mathbf{T}$. If only one receiver position is used, it is not possible to distinguish the correct order of detections and assign submatrix $\mathbf{D}_{p o}$ to the corresponding $\mathbf{T}_{p o}$. This ambiguity is solved by adding another dimension to matrix $\mathbf{O}$, including all permutations of transmitter order. This is the same way the matrix $\mathbf{D}$ is modified. The elements of matrix $\mathbf{O}$ are now given by:

$$
O_{n k p o}=D_{n k p o}-T_{n p o}
$$

where $k$ is a transmitter permutation index. If more than one measurement is performed in different positions, only one permutation $k$ results in the same values in all submatrices $\mathbf{O}_{n}$. This permutation not only gives us relative offset values in the measured SFN, but also matches them to the individual transmitters. This knowledge can be used to correct arbitrary position measurement using this network. The relative offsets slightly differ in the individual measurements 
because of many influences. The resulting relative offset values from the individual measurements are averaged to reduce measurement inaccuracies. At this moment all is settled, and the position can be evaluated.

\section{Position Estimation}

For the TDoA positioning scenario, the NewtonRaphson iterative algorithm, similar to the ToA method described in [16], is used. This modification can be found in [8]. As noted earlier, the time differences of arrival from the individual transmitters are obtained from the detection algorithm. This leads to the TDoA positioning method.

The automatic detection algorithm described in Sec. 5 provide a local time of the individual detections. For the TDoA positioning method is necessary only the time differences between individual detections. Hence, we do not need to solve time offset between the local time and the global timing in the SFN.

The first step of the TDoA algorithm is a conversion of the arrival time differences to the distance differences by multiplying by the speed of light. The distance differences are organized into the matrix with coefficients given by:

$$
d_{i j}=\left\|\mathbf{r}_{u}-\mathbf{r}_{i}\right\|-\left\|\mathbf{r}_{u}-\mathbf{r}_{j}\right\|+\epsilon_{12} .
$$

Here $\mathbf{r}_{u}$ is a vector containing receiver coordinates, $\mathbf{r}_{i}$ is a vector containing coordinates of the $i^{\text {th }}$ transmitter, and the additive error $\epsilon_{12}$ is considered in the measured time differences.

For the iterative solver the variables are separated into predictive $\left(\mathbf{r}_{u \mathrm{p}}\right)$ and corrective $\left(\mathbf{r}_{u \mathrm{c}}\right)$ parts. Applying the Taylor approximation of the $1^{\text {st }}$ order to distances between the receiver and transmitters results in:

$$
\left\|\mathbf{r}_{u \mathrm{p}}+\mathbf{r}_{u \mathrm{c}}-\mathbf{r}_{i}\right\| \approx\left\|\mathbf{r}_{u \mathrm{p}}-\mathbf{r}_{i}\right\|+\frac{\left(\mathbf{r}_{u \mathrm{p}}-\mathbf{r}_{i}\right)}{\left\|\mathbf{r}_{u \mathrm{p}}-\mathbf{r}_{i}\right\|} \mathbf{r}_{u \mathrm{c}}
$$

where the second part of the equation contains the unitary direction vector defined as:

$$
\mathbf{1}_{u i}=\frac{\left(\mathbf{r}_{u \mathrm{p}}-\mathbf{r}_{i}\right)}{\left\|\mathbf{r}_{u \mathrm{p}}-\mathbf{r}_{i}\right\|} .
$$

Now, using the direction vector (15) and the corrective part of the difference matrix d given by (13), it is possible to write the corrective part as:

$$
\mathbf{d}_{\mathrm{c}}=\left[\begin{array}{c}
\mathbf{1}_{u i}-\mathbf{1}_{u j} \\
\vdots \\
\mathbf{1}_{u i}-\mathbf{1}_{u j}
\end{array}\right] \mathbf{r}_{u \mathrm{c}}=\mathbf{G r}_{u \mathrm{c}} .
$$

The geometry matrix $\mathbf{G}$ is not generally square. For that reason, pseudo-inversion defined by:

$$
\mathbf{r}_{u \mathrm{c}}=\left(\mathbf{G}^{\mathrm{T}} \mathbf{G}\right)^{-1} \mathbf{G} \mathbf{d}_{\mathrm{c}}
$$

is used to solve the corrective part while respecting the least square criterion. This equation is used to estimate receiver position iteratively. In the beginning, the arbitrary initial point in the vicinity of the network is used as a position prediction $\mathbf{r}_{u \mathrm{p}}$. Then, the correction $\mathbf{r}_{u \mathrm{c}}$ is computed using (17), and the predicted position is corrected. This algorithm is repeated until the Euclidean norm of the correction vector is lower than a given threshold. This algorithm quickly converges to the correct solution after several iterations. Typically, the Euclidean norm of the correction vector after seven iterations is in order of $0.1 \mathrm{~m}$ and after eight iterations in order of $1 \times 10^{-7} \mathrm{~m}$.

\section{Experimental Verification with a Real DVB-T Broadcast}

At first, we need to note that the performance of the achieved results in this experimental verification of our proposed method cannot be compared with the literature because no other test, including automatic detection and position evaluation, has been published. Furthermore, the relevant papers seem to suffer from lack of the SFN offset solution.

The method for utilizing a DVB-T network for positioning was verified by the experimental measurement of the real signal in Prague (Czech Republic). Channel 42, with a center frequency of $642 \mathrm{MHz}$ and consisting of three transmitters working in the SFN, is an ideal choice to demonstrate the designed positioning system. Transmitters in this network are deployed nearly in one line across the city. At the north, the Ládví $\left(\mathbf{T x}_{1}\right)$ transmitter transmits with $20 \mathrm{~kW}$ Effective Radiated Power (ERP). Further south from $\mathbf{T} \mathbf{x}_{1}$ is the Olšanská transmitter $\left(\mathbf{T x}_{\mathbf{2}}\right)$ with $10 \mathrm{~kW}$ ERP, and the greater southern area is covered by the Novodvorská transmitter $\left(\mathbf{T x}_{\mathbf{3}}\right)$ with $5 \mathrm{~kW}$ ERP. The GPS coordinates of the individual transmitters are shown in Tab. 1.

The measured points in this experiment are designed to minimize the influence of signal propagation, such as diffraction and multipath, to lower transmitter offset estimation error. Positions, with a free line of sight from receiver to almost all of the transmitters, have been selected for this experiment. Their coordinates are shown in Tab. 1. Figure 9 illustrates the overview of the experimental scenario. Red points mark the position of the transmitters and the measurement spots are blue.

The Software-define Radio (SDR) ETTUS USRP N210, featuring a dual 14-bit Analog-to-Digital Converter (ADC), with a sampling frequency $100 \mathrm{MS} / \mathrm{s}$ and a daughterboard UBX $10-6000 \mathrm{MHz} \mathrm{Rx} / \mathrm{Tx}$, is used as the receiver. It is possible to connect it directly with MATLAB to download sampled data. A marine omnidirectional Glomex TV antenna is used as a receiver antenna. The block diagram of the experimental receiver is shown in Fig. 8. 


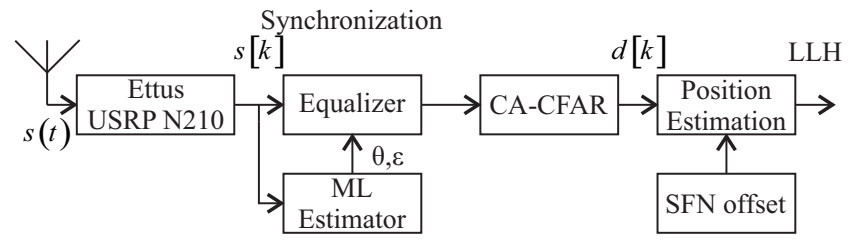

Fig. 8. The block diagram of the experimental receiver.

\begin{tabular}{|l|c|c|c|}
\hline Transmitter & Latitude $^{\circ}$ & Longitude $^{\circ}$ & $\begin{array}{c}\text { Altitude } \\
\text { [m a.s.1.] }\end{array}$ \\
\hline $\mathbf{T x}_{\mathbf{1}}$ (Ládví) & 50.13642 & 14.46528 & 359 \\
$\mathbf{T x}_{\mathbf{2}}$ (Olšanská) & 50.08352 & 14.46910 & 250 \\
$\mathbf{T x}_{\mathbf{3}}$ (Novodvorská) & 50.01638 & 14.45111 & 305 \\
\hline Receiver & Latitude $^{\circ}$ & Longitude $^{\circ}$ & $\begin{array}{c}\text { Altitude } \\
\text { [m a.s.1.] }\end{array}$ \\
\hline $\mathbf{R x}$ 1 (Parukářka) & 50.08510 & 14.46022 & 300 \\
$\mathbf{R x}_{2}$ (Strahov) & 50.08048 & 14.39566 & 330 \\
$\mathbf{R x}_{\mathbf{1}}$ (Suchdol) & 50.13175 & 14.38581 & 275 \\
\hline
\end{tabular}

Tab. 1. The coordinates of the DVB-T transmitters and the measured positions.

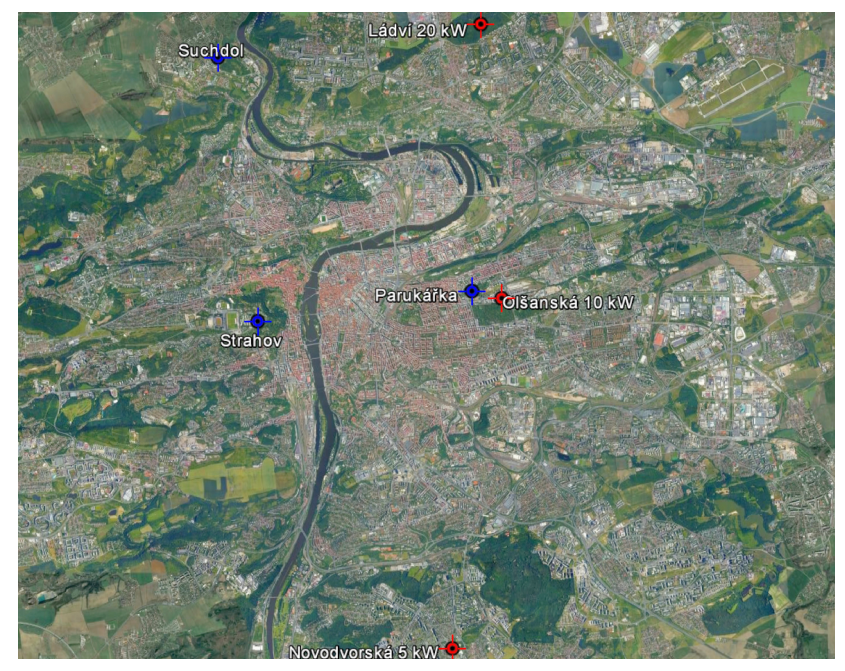

Fig. 9. The overview of the experiment scenario.

Two measurements were performed at every position and every time with a sampling frequency $10 \mathrm{MHz}$, with the duration of the record corresponding to 10 OFDM symbols. The experiment tends to estimate offset in the SFN and to use it to evaluate the correct position of the receiver. The result of the performed experiment is presented later in this section.

The measured data are processed with the algorithm that first finds the duration of the guard interval and then the beginning of the OFDM symbol before performing time and frequency synchronization of the signal. When the actual pattern of the SP sequence is found, it is used to choose the correct impulse response of the mismatched filter which is pre-generated according to the bandwidth used with the sampled data. The signal is then separated into individual OFDM symbols, and the guard interval is omitted. Symbols are filtered using a corresponding mismatched filter and added together in absolute value. The filtration is performed only on the interval corresponding to SP sequence duration. At this point the pre-processed data sequence is interpolated and processed with the CA-CFAR algorithm to obtain detections.

With the ambiguity problem solved, the individual detections are assigned to the corresponding transmitters using additional information from a GPS receiver. Since the DVB-T-based positioning system is intended as a supplementary system in hard conditions, it is possible to assume that the receiver position is roughly known from the primary navigation system, so when an outage occurs, the last-known position from the primary system is used to solve an ambiguity problem and identify the DVB-T transmitters.

A visualization of a CA-CFAR algorithm for one experimental measurement on every position is shown in Figs. 10, 11 and 12. Filtered data are shown by the red line and the threshold estimated in CA-CFAR is blue. It can be seen that the threshold rapidly drops around the peak in data. When data are shifted, the peak is situated in the middle window of the shift register and is not included in the threshold estimation. When the signal exceeds the threshold, detection occurs. Following a successful detection, the threshold remains high until the peak is situated in the left window of the shift register. Thanks to this, many false detections caused by multipath are suppressed.

The time offset in the SFN must be known before the position can be evaluated. The algorithm described in Sec. 6 is applied. Then, all permutations of the relative offsets are evaluated and stored in matrix $\mathbf{O}$.

This matrix can be divided into submatrices corresponding to the individual experiments where only one line in each submatrix contains the same offset values, which is the correction for the SFN. The obtained detections from the data are imported into the SFN offset measurement algorithm to obtain averaged relative offsets. Table 2 shows three submatrices of $\mathbf{O}_{\mathbf{n}}$, containing all offset permutations for the $n^{\text {th }}$ experiment. Values in each line are sorted, and since the obtained offsets are relative, the first value is zero.

In each $\mathbf{O}_{n}$ evaluated from the six performed experiments, we can find one permutation $(k)$ with similar values. In the submatrices are those similar values in the second line. No other line consists of similar values. The relative offset obtained from the experiments are averaged and used as an offset correction. It was possible to estimate relative offsets $0.0897 \mu$ s and $41.8472 \mu$ s for the $\mathbf{T} \mathbf{x}_{\mathbf{1}}$ and $\mathbf{T} \mathbf{x}_{\mathbf{2}}$, respectively. Those values are relative to the $\mathbf{T} \mathbf{x}_{\mathbf{3}}$ since the absolute offset value cannot be obtained using this method.

At this point it is possible to start positioning from the data in this SFN. The output of the positioning algorithm using the correction of a transmitters' delay is shown in Tab. 3. The value of the Horizontal Dilution of Precision (HDoP) can be seen in the fourth column. This can be easily obtained from geometry matrix $\mathbf{G}$ defined in (16) as follows:

$$
\text { HDOP }=\sqrt{\operatorname{tr}\left(\left(\mathbf{G}^{\mathrm{T}} \mathbf{G}\right)^{-1}\right)}
$$




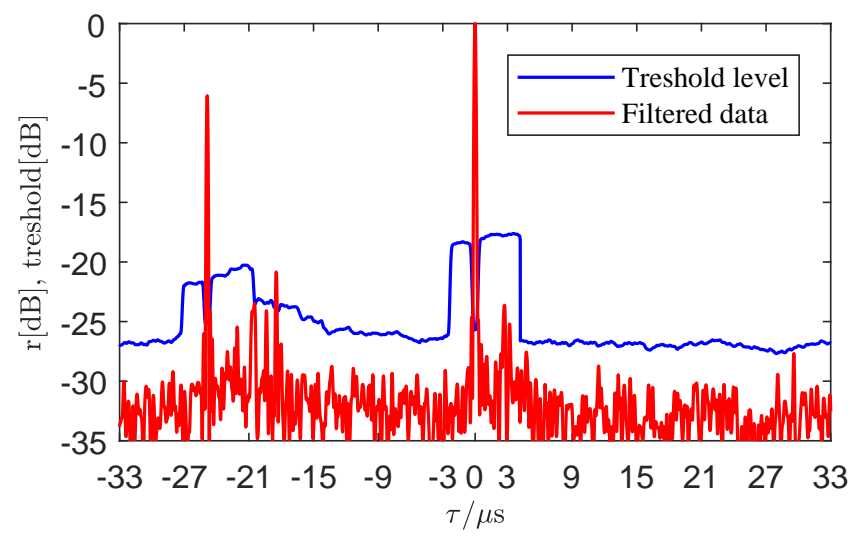

Fig. 10. The output of the CA-CFAR algorithm for the experiment at $\mathbf{R} \mathbf{x}_{\mathbf{1}}$.

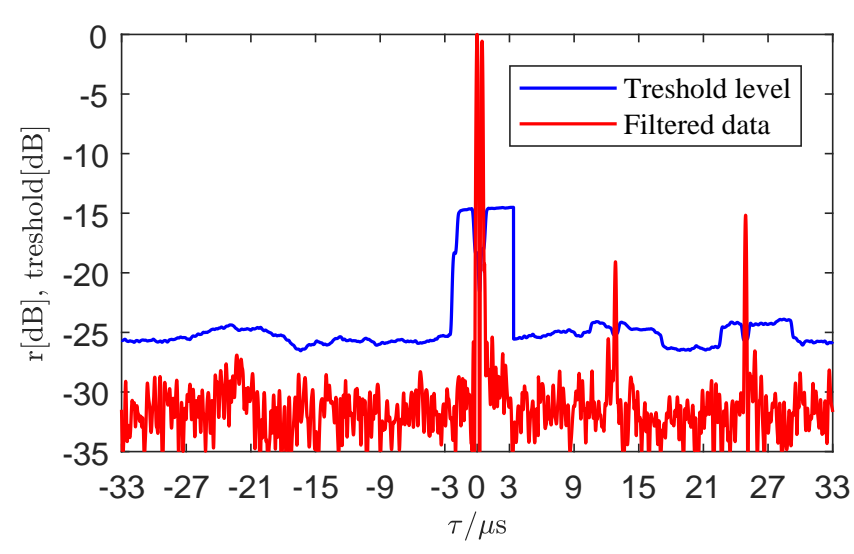

Fig. 11. The output of the CA-CFAR algorithm for the experiment at $\mathbf{R} \mathbf{x}_{2}$.

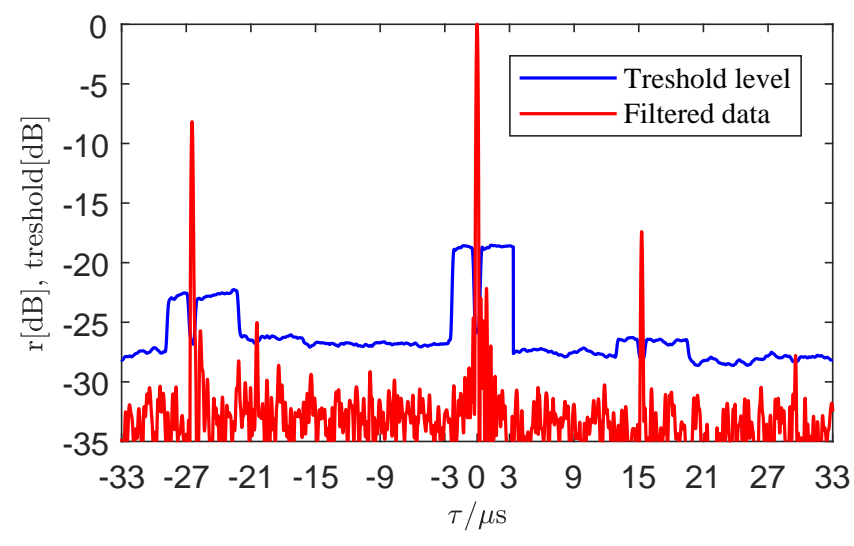

Fig. 12. The output of the CA-CFAR algorithm for the experiment at $\mathbf{R} \mathbf{x}_{3}$.

The aim of this experiment was to confirm the assumption that there are constant time offsets applied to DVB-T transmitters and that it is possible to estimate the relative values of these offsets and use them to evaluate the correct position. Even in this small sample, it is possible to determine that HDoP have a major influence on position evaluation as it changes rapidly within the area covered by the SFN signal.

\begin{tabular}{|l|l|l|l|}
\hline $\mathrm{k}$ & \multicolumn{3}{|c|}{$\mathbf{O}_{1}\left(\mathbf{R x _ { 1 } )}[\mu \mathrm{s}]\right.$} \\
\hline 1 & 0 & 12.9066 & 48.2440 \\
2 & 0 & 00.1066 & 41.8440 \\
3 & 0 & 29.7840 & 31.3666 \\
4 & 0 & 18.3534 & 23.2700 \\
5 & 0 & 16.9840 & 24.9666 \\
6 & 0 & 10.4774 & 11.9534 \\
\hline
\end{tabular}$\quad$\begin{tabular}{|l|l|l|l|}
$\mathrm{k}$ & \multicolumn{3}{|c|}{$\mathbf{O}_{2}\left(\mathbf{R x}_{\mathbf{2}}\right)[\mu \mathrm{s}]$} \\
\hline 1 & 0 & 01.0540 & 42.3130 \\
2 & 0 & 00.1140 & 41.8430 \\
3 & 0 & 10.0864 & 33.2806 \\
4 & 0 & 09.5024 & 32.1127 \\
5 & 0 & 09.1464 & 32.8106 \\
6 & 0 & 08.5624 & 31.6427 \\
\hline
\end{tabular}

\begin{tabular}{|l|l|l|l|}
\hline $\mathrm{k}$ & \multicolumn{3}{|c|}{$\mathbf{O}_{3}\left(\mathbf{R x}_{\mathbf{3}}\right)[\mu \mathrm{s}]$} \\
\hline 1 & 0 & 52.9687 & 68.3195 \\
2 & 0 & 00.0487 & 41.8595 \\
3 & 0 & 45.2728 & 76.0153 \\
4 & 0 & 18.7641 & 22.9980 \\
5 & 0 & 07.6472 & 57.2025 \\
6 & 0 & 30.6939 & 34.1559 \\
\hline
\end{tabular}

Tab. 2. The evaluated offset values in sub-matrices $\mathbf{O}_{\mathbf{n}}$.

\begin{tabular}{|l|c|c|c|c|}
\hline Receiver & Latitude $^{\circ}$ & Longitude $^{\circ}$ & HDoP & Err [m] \\
\hline $\mathbf{R x}_{1}$ I & 50.08507 & 14.46034 & 0.8 & 9 \\
$\mathbf{R x}_{1}$ II & 50.08510 & 14.46018 & 0.8 & 3 \\
$\mathbf{R x}_{\mathbf{2}}$ I & 50.08034 & 14.39604 & 1.7 & 30 \\
$\mathbf{R x}_{\mathbf{2}}$ II & 50.08031 & 14.39587 & 1.7 & 22 \\
$\mathbf{R x}_{3}$ I & 50.13175 & 14.38594 & 3.8 & 9 \\
$\mathbf{R x}_{3}$ II & 50.13123 & 14.38721 & 3.7 & 115 \\
\hline
\end{tabular}

Tab. 3. The coordinates obtained from measurements.

\section{DVB-T2 Signal Modification}

The DVB-T gone through a major revision and many parts were updated to provide even better properties. Moreover, it offers the high definition television standard. This led to the second generation of the digital video broadcasting DVB-T2. Since the proposed positioning algorithm is based solely on pilot signaling, we need to look closely to the changes made in pilot signaling to be able to decide if the current work is compatible with the DVB-T2 system.

The first major change in DVB-T2 is that values from PRBS sequence are no longer directly mapped to pilot subcarriers. The PRBS sequence is combined with the frame level PN-sequence using exclusive-or gate and the output is used to evaluate values of pilot subcarriers [3].

The next difference is the SP and CP locations. The SPs are now mapped according to eight possible pilot patterns (PP1-PP8). Not all of the possible patterns are available for a given FFT size and the guard interval duration. The $\mathrm{CP}$ are now given by $6 \mathrm{CP}$ groups. The number of groups being used is given by the actual FFT mode. Only one CP group is used in $1 \mathrm{~K}$ FFT mode and all six groups are used in $32 \mathrm{~K}$ mode.

This change requires to use this new pilot signaling for positioning system based on DVB-T2. However, the main principle of automatic detection algorithm and position estimation remain unchanged. Even the resolution is not negatively affected since the frequency bandwidth remains unchanged. What can be affected, both positively and negatively, is the maximal unambiguous relative distance caused by pilot spacing. 
For example, if the PP3 is used in the $8 \mathrm{~K}$ mode the pilot is inserted every $24^{\text {th }}$ OFDM symbol and the unambiguous relative distance is half the distance of the DVB-T $8 \mathrm{~K}$ mode, where the SP symbol is every $12^{\text {th }}$ subcarrier. However, if the PP2 is used the unambiguous distance remain unchanged.

The DVB-T2 signal synchronization method would change. We would need to receive the L1 signaling data to obtain actual parameters of a transmission. Then it is possible to perform channel estimation using either least square or minimum mean square error estimation. Detailed analysis of pilot patterns and channel estimation of the DVB-T2 channel can be found in [17].

\section{Conclusion}

This paper focuses on improving the properties of a DVB-T based positioning system. The described methods allow creating a standalone receiver which can evaluate the position autonomously. This system can be implemented into the multisensor positioning unit to increase its overall properties.

The pre-processing method greatly improves time domain properties, especially the sidelobe ratio which is now $23 \mathrm{~dB}$, in contrast to $13 \mathrm{~dB}$ when mismatched filtration is not used. This enables the detector to distinguish extremely close detections with spacing under $0.5 \mu$ s and suppress false alarms caused by sidelobes of the filtered signal, a useful addition for scenarios featuring a similar signal time of arrival from multiple transmitters.

The suggested noncoherent CA-CFAR detection method has been shown to work for detection of time differences of signal arrival in the DVB-T network. This detection algorithm enables the fully automatic evaluation of the receiver position even in a real multipath scenario. The noncoherent averaging method can improve SNR and the properties of weak signal level scenarios. This is a significant step toward an automatic positioning system in DVB-T networks.

The experiments revealed a problem with artificially added time offsets in SFN where transmitters appear to be at a greater distance than they are. Every emitter in the network can have a different value of the offset and cause incorrect position estimations. A method to measure the relative value of those offsets to suppress its influence during positioning has been developed.

All described methods are utilized in Sec. 8 to proof its functionality. It was possible to estimate relative offsets in the tested SFN and use them to evaluate a correct position. We performed experiments in three locations in Prague (Czech Republic) to evaluate time offset correction and position. However, more experiments are needed to study system precision, but we have evaluated position with an expected error in the order of ones to tens of meters depending on the DoP estimation.
It is possible to use a DVB-T signal as a supplementary system for a GNSS positioning system in so-called hard conditions when a satellite system cannot provide sufficient service. This can occur in indoor scenarios where a high-power DVB-T signal is strong enough for positioning and a GNSS signal cannot be used. Further testing in hard conditions and indoor scenarios are required to improve the presented positioning system and to study its capabilities.

\section{Acknowledgments}

The work was supported by the CTU grant No. SGS17/182/OHK3/3T/13.

\section{References}

[1] KAPLAN, E., HEGARTY, C. Understanding GPS: Principles and Applications. 2nd ed., rev. Norwood (US): Artech House, 2005. ISBN: 9781580538947

[2] Digital Video Broadcasting (DVB); Framing Structure, Channel Coding and Modulation for Digital Terrestrial Television DVB-T. ETSI EN 300744 1.6.2, Oct. 2015

[3] Digital Video Broadcasting (DVB); Frame Structure Channel Coding and Modulation for a second Generation Digital Terrestrial Television Broadcasting System (DVB-T2). ETSI EN 302755 1.1.1, 2009

[4] Digital Video Broadcasting (DVB); DVB Mega-Frame for Single Frequency Network (SFN) Synchronization. ETSI TS 101191 1.4.1, 2004

[5] RICNY, V. Single frequency networks (SFN) in digital terrestrial broadcasting. Radioengineering, 2007, vol. 16, no. 4, p. 2-6. ISSN: $1210-2512$.

[6] CHEN, L., YANG, L.L., YAN, J., et al. Joint wireless positioning and emitter identification in DVB-T single frequency networks. IEEE Transactions on Broadcasting, 2017, vol. 63, no. 3, p. 577-582. ISSN: 0018-9316. DOI: 10.1109/TBC.2017.2704422

[7] CHEN, L., JULIEN, O., THEVENON, P., et al. TOA estimation for positioning with DVB-T signals in outdoor static tests. IEEE Transactions on Broadcasting, 2015, vol. 61, no. 4, p. 625-638. ISSN: 0018-9316. DOI: 10.1109/TBC.2015.2465155

[8] NAVRATIL, V., KARASEK, R., VEJRAZKA, F. Position estimate using radio signals from terrestrial sources. In IEEE/ION Position, Location and Navigation Symposium (PLANS). Savannah (Georgia), 2016, p. 799-806. DOI: 10.1109/PLANS.2016.7479775

[9] KARASEK, R. Amendment of GNSS Systems in Hard Conditions by 'Opportunity signals'. M.S. thesis, Faculty of Electrical Engineering, Czech Technical University in Prague, 2017. [Online] Available at: https://dspace.cvut.cz/handle/10467/68453?locale-attribute=en

[10] CHEN, L., THEVENON, P., SECO-GRANADOS, G., et al. Analysis on the TOA tracking with DVB-T signals for positioning. IEEE Transactions on Broadcasting, 2016, vol. 62, no. 4, p. 957-961. ISSN: 0018-9316. DOI: 10.1109/TBC.2016.2606939

[11] SKOLNIK, M. I. Radar Handbook. 3rd ed., rev. New York (US): McGraw Hill, 2008. ISBN: 9780071485470

[12] VAN DE BEEK,J. J., SANDELL, M., BORJESSON, P. O. ML estimation of time and frequency offset in OFDM systems. IEEE Transactions on Signal Processing, 1997, vol. 42, no. 7, p. 1800-1805. ISSN: 1053-587X. DOI: 10.1109/78.599949 
[13] CHEN, C. Y., WANG, Y. T., HUNG, Y. H. Time-Frequency Correlation-Based Synchronization for Coherent OFDM Receiver. U.S. Patent 11/153,105. 2006

[14] PRABHU, K. M. M. Window Functions and Their Applications in Signal Processing. 1st ed., rev. Boca Raton (US): CRC Press, 2013. ISBN: 9781466515840

[15] KAY, S. M. Fundamentals of Statistical Signal Processing, Volume II: Detection Theory. 1st ed., rev. Upper Saddle River (US): PrenticeHall PTR, 1998. ISBN: 9780135041352

[16] MISRA, P., ENGE, P. Global Positioning System: Signals, Measurements, and Performance. 1st ed., rev. Lincoln (US): Ganga-Jamuna Press, 2001. ISBN: 9780970954404

[17] FU, L., SUN, S., JING, X., et al. Analysis of pilot patterns and channel estimation for DVB-T2. In Proceedings of 2nd IEEE International Conference on Network Infrastructure and Digital Content. Beijing (China), 2010, p. 609-613. DOI: 10.1109/ICNIDC.2010.5657853
About the Authors ...

Rostislav KARASEK received the Ing. degree in wireless communications from the Faculty of Electrical Engineering, Czech Technical University in Prague, Czech Republic, in 2017, where he is currently pursuing Ph.D. degree. His research interest includes the study of electromagnetic field propagation and signal processing enabling refinement of indoor positioning systems and through-wall sensing.

Frantisek VEJRAZKA received the Ing. degree in 1965, the CSc. in 1972, associate professor in 1982 and professor in 1996, all from the Faculty of Electrical Engineering, Czech Technical University in Prague, Czech Republic, where he is currently the head of the navigation research group, dep. of Radioelectronics. His research interests include GNSS and sensor fusion for outdoor and indoor positioning. 